Bull. Korean Math. Soc. 50 (2013), No. 4, pp. 1157-1171

http://dx.doi.org/10.4134/BKMS.2013.50.4.1157

\title{
VALUE DISTRIBUTION AND UNIQUENESS ON $q$-DIFFERENCES OF MEROMORPHIC FUNCTIONS
}

\author{
ZHI-Bo HuANG
}

\begin{abstract}
In this paper, by using the $q$-difference analogue of lemma on the logarithmic derivative lemma to re-establish some estimates of Nevanlinna characteristics of $f(q z)$, we deal with the value distribution and uniqueness of certain types of $q$-difference polynomials.
\end{abstract}

\section{Introduction}

In this paper, we assume that the reader is familiar with the standard symbols and fundamental results of Nevanlinna theory, such as the proximity function $m(r, f)$, counting function $N(r, f)$, characteristic function $T(r, f)$ for a meromorphic function $f(z)$ in the complex plane (see e.g. $[7,14]$ ). We also use $\bar{N}_{(2}\left(r, \frac{1}{f}\right)$ to denote the counting function of zeros of $f(z)$ such that the multiple zeros are counted once and the simple zeros are not counted in $\{z:|z| \leq r\}$. We now recall that a meromorphic function $a(z)$ is said to be a small function of $f(z)$ if $T(r, a)=S(r, f)$, where $S(r, f)$ is used to denote any quantity satisfying $S(r, f)=o(\{T(r, f)\}$ as $r \rightarrow \infty$, possibly outside of a set of finite logarithmic measure, furthermore, possibly outside of a set of logarithmic density 0 , i.e., outside of a set $E$ such that $\lim _{r \rightarrow \infty} \int_{[1, r] \cap E} \frac{d t}{t} / \log r=0$. The family of all small functions related to $f(z)$ is denoted by $\mathscr{F}(f)$.

Recently, a number of fundamental results on difference operators and difference polynomials have been derived. For examples, the difference analogue of lemma on the logarithmic derivative [2, 5], the difference counterpart of Clunie and Mohon'ko lemma [5, 9], Nevanlinna characteristics of $f(z+c)$ for $c \in \mathbb{C} \backslash\{0\}$ in the complex plane [2] and Nevanlinna theory to difference operators, especially the difference analogue of the second main theorem [6]. Using these results, the value distribution and uniqueness of difference operators and difference polynomials of meromorphic functions have been dealt with in the

Received July 11, 2012.

2010 Mathematics Subject Classification. 30D35, 39A10.

Key words and phrases. uniqueness, $q$-difference polynomial, characteristic function, sharing value.

Research supported by National Natural Science Foundation of China(Nos.11171119, 11171121). 
past five years (see e.g. $[3,4,8,10,11]$ ). However, there are only few papers concerning with the value distribution and uniqueness of $q$-difference operators and $q$-difference polynomials (see $[12,16]$ ).

The purpose of this paper is to study the value distribution and uniqueness of $q$-differences of meromorphic function of zero order. The main tool is to use the $q$-difference analogue of lemma on the logarithmic derivative [1] to reestablish some estimates on the Nevanlinna characteristics of $f(q z)$, which are somewhat different from Nevanlinna characteristics of $f(q z)$ obtained by Zhang and Korhonen in [16].

This paper is organized as follows. In Section 2, we present some results on value distribution of $q$-difference polynomials of meromorphic functions of zero order. In Section 3, we investigate uniqueness of $q$-difference polynomials of meromorphic functions of zero order.

\section{Value distribution of $q$-difference polynomials}

Laine and Yang [10] investigated the value distribution of difference products of entire functions and obtained the following result.

Theorem 2.A ([10, Theorem 2]). Let $f(z)$ be a transcendental entire function of finite order, and $c$ be a nonzero complex constant. Then for $n \geq 2, f(z)^{n} f(z+$ c) assumes every nonzero value $a \in \mathbb{C}$ infinitely often.

Subsequently, a parallel result for the $q$-difference case has been proved in $[16]$.

Theorem 2.B ([16, Theorem 4.1]). Let $f(z)$ be a transcendental meromorphic (resp. entire) function of zero order and $q$ be nonzero complex constant. Then for $n \geq 6$ (resp. $n \geq 2), f(z)^{n} f(q z)$ assumes every nonzero value a $\in \mathbb{C}$ infinitely often.

In addition, we also recall the following related result.

Theorem 2.C ([16, Theorem 4.3]). Let $f(z)$ be a transcendental meromorphic (resp. entire) function of zero order and $q$ be nonzero complex constant. Then for $n \geq 6$ (resp. $n \geq 2), f(z)^{n}(f(z)-1) f(q z)$ assumes every nonzero value $a \in \mathbb{C}$ infinitely often.

In this section, we will establish an improvement of Theorem 2.B and Theorem 2.C, which is stated as follows.

Theorem 2.1. Let $f(z)$ be a transcendental meromorphic (resp. entire) function of zero order and $q$ be nonzero complex constant, and let $P(z)=a_{n} z^{n}+$ $a_{n-1} z^{n-1}+\cdots+a_{1} z+a_{0}$ be a nonconstant polynomial with constant coefficients $a_{0}, a_{1}, \ldots, a_{n-1}, a_{n}(\neq 0)$, and $m$ be the number of the distinct zeros of $P(z)$. Then for $n>2 m+3$ (resp. $n>m), P(f(z)) f(q z)-a(z)$ has infinitely many zeros, where $a(z) \in \mathscr{F}(f) \backslash\{0\}$.

The restriction in Theorem 2.1 to $a(z) \in \mathscr{F}(f) \backslash\{0\}$ is essential. 
Example 2.1. Let $q \in \mathbb{C}$ such that $0<|q|<1$. The $q$-Gamma function $\Gamma_{q}(x)$ is defined by

$$
\Gamma_{q}(x):=\frac{(q ; q)_{\infty}}{\left(q^{x} ; q\right)_{\infty}}(1-q)^{1-x}
$$

where $(a ; q)_{\infty}=\Pi_{k=0}^{\infty}\left(1-a q^{k}\right)$. By defining

$$
\gamma_{q}(z):=(1-q)^{x-1} \Gamma_{q}(x), z=q^{x},
$$

and $\gamma_{q}(0):=(q ; q)_{\infty}$, we see that $\gamma_{q}(z)$ is meromorphic of zero order with no zero. By taking $P(z)=z$ and $f(z)=\gamma_{q}(z)$. If $a(z) \equiv 0$, then $P(f(z)) f(q z)-$ $a(z)=\gamma_{q}(z) \cdot \gamma_{q}(q z)$ has no zero.

Example 2.2. The zero order growth restriction in Theorem 2.1 can not be extended to finite order. This can be seen by taking $P(z)=z^{n}+1, f(z)=e^{z}$ and $q=-n$. Then $P(f(z)) f(q z)-1$ has no zero.

In order to prove Theorem 2.1, we need some preliminaries as follows.

Lemma 2.1 ([1, Lemma 5.2]). If $T: \mathbb{R}^{+} \rightarrow \mathbb{R}^{+}$is a piecewise continuous increasing function such that

$$
\lim _{r \rightarrow \infty} \frac{\log T(r)}{\log r}=0
$$

then the set

$$
E:=\left\{r: T\left(C_{1} r\right) \geq C_{2} T(r)\right\}
$$

has logarithmic density 0 for all $C_{1}>1$ and $C_{2}>1$.

Lemma 2.2. Let $f(z)$ be a nonconstant meromorphic function of zero order, and $q \in \mathbb{C} \backslash\{0\}$. Then

$$
\begin{aligned}
& N\left(r, \frac{1}{f(q z)}\right) \leq N\left(r, \frac{1}{f(z)}\right)+S(r, f), \\
& N(r, f(q z)) \leq N(r, f(z))+S(r, f), \\
& \bar{N}\left(r, \frac{1}{f(q z)}\right) \leq \bar{N}\left(r, \frac{1}{f(z)}\right)+S(r, f), \\
& \bar{N}(r, f(q z)) \leq \bar{N}(r, f(z))+S(r, f),
\end{aligned}
$$

on a set of logarithmic density 1.

Proof. We will use the similar method used in [16]. Here, we only prove the case $|q|>1$. By a simple geometric observation, we obtain

$$
N\left(r, \frac{1}{f(q z)}\right) \leq N\left(|q| r, \frac{1}{f(z)}\right) .
$$

Since the order of $f(z)$ is zero, we conclude from Lemma 2.1 that,

$$
N\left(|q| r, \frac{1}{f(z)}\right) \leq N\left(r, \frac{1}{f(z)}\right)+S(r, f),
$$


on a set of logarithmic density 1 . Therefore,

$$
N\left(r, \frac{1}{f(q z)}\right) \leq N\left(r, \frac{1}{f(z)}\right)+S(r, f),
$$

on a set of logarithmic density 1 .

Similarly, we can prove the remainders. Here, we omit their proofs.

Now, we recall the $q$-difference analogue of lemma on the logarithmic derivative as follows.

Lemma 2.3 ([1, Theorem 1.2]). Let $f(z)$ be a nonconstant zero order meromorphic function, and $q \in \mathbb{C} \backslash\{0\}$. Then

$$
m\left(r, \frac{f(q z)}{f(z)}\right)=o(T(r, f))
$$

on a set of logarithmic density 1 .

Lemma 2.4. Let $f(z)$ be a nonconstant meromorphic function of zero order, and $q \in \mathbb{C} \backslash\{0\}$. Then

$$
T(r, f(q z)) \leq T(r, f(z))+S(r, f)
$$

on a set of logarithmic density 1.

Proof. By Lemma 2.2 and Lemma 2.3, we obtain

$$
\begin{aligned}
T(r, f(q z)) & =m(r, f(q z))+N(r, f(q z)) \\
& \leq m\left(r, \frac{f(q z)}{f(z)}\right)+m(r, f(z))+N(r, f(z))+S(r, f) \\
& =T(r, f(z))+S(r, f)
\end{aligned}
$$

on a set of logarithmic density 1 .

Remark 2.1. In [16], the authors showed that the conclusion in Lemma 2.4 holds on a set of lower logarithmic density 1.

Lemma 2.5. Let $f(z)$ be an entire function of zero order and $q$ be nonzero constant, and let $P(z)=a_{n} z^{n}+a_{n-1} z^{n-1}+\cdots+a_{1} z+a_{0}$ be a nonconstant polynomial with constant coefficients $a_{0}, a_{1}, \ldots, a_{n-1}, a_{n}(\neq 0)$. Then

$$
T(r, P(f(z)) f(q z))=T(r, P(f(z)) f(z))+S(r, f)
$$

on a set of logarithmic density 1 .

Proof. Since $f(z)$ is entire of zero order, we obtain, by Lemma 2.3,

$$
\begin{aligned}
T(r, P(f(z)) f(q z)) & =m(r, P(f(z)) f(q z)) \\
& \leq m(P(f(z)) f(z))+m\left(r, \frac{f(q z)}{f(z)}\right)+S(r, f) \\
& =T(r, P(f(z)) f(z))+S(r, f)
\end{aligned}
$$


on a set of logarithmic density 1. Similarly, we also have

$$
T(r, P(f(z)) f(z)) \leq T(r, P(f(z)) f(q z))+S(r, f)
$$

on a set of logarithmic density 1 . Therefore,

$$
T(r, P(f(z)) f(q z))=T(r, P(f(z)) f(z))+S(r, f)
$$

on a set of logarithmic density 1 .

Now, we give a proof of Theorem 2.1 completely.

Proof of Theorem 2.1. Suppose that $P(f(z)) f(q z)-a(z)$ has finitely many zeros only. If $f(z)$ is meromorphic of zero order, then we may apply the ValironMohon'ko lemma, Nevanlinna main theorems, Lemma 2.4 and Lemma 2.2 to obtain

$$
\begin{aligned}
n T(r, f)+S(r, f)= & T(r, P(f(z)) \\
\leq & T(r, P(f(z)) f(q z))+T\left(r, \frac{1}{f(q z)}\right)+S(r, f) \\
\leq & T(r, f(z))+\bar{N}(r, P(f(z)) f(q z))+\bar{N}\left(r, \frac{1}{P(f(z)) f(q z)}\right) \\
& +\bar{N}\left(r, \frac{1}{P(f(z)) f(q z)-a(z)}\right)+S(r, f) \\
\leq & T(r, f(z))+\bar{N}(r, P(f))+\bar{N}(r, f(q z)) \\
& +\bar{N}\left(r, \frac{1}{P(f(z))}\right)+\bar{N}\left(r, \frac{1}{f(q z)}\right)+S(r, f) \\
\leq & T(r, f(z))+m \bar{N}(r, f(z))+\bar{N}(r, f(z)) \\
& +m \bar{N}\left(r, \frac{1}{f(z)}\right)+\bar{N}\left(r, \frac{1}{f(z)}\right)+S(r, f) \\
\leq & (2 m+3) T(r, f)+S(r, f),
\end{aligned}
$$

on a set of logarithmic density 1 , contradicting $n>2 m+3$.

If, on the other hand, $f(z)$ is entire of order zero, then

$$
\begin{aligned}
T(r, P(f(z)) f(q z)) \leq & \bar{N}\left(r, \frac{1}{P(f(z)) f(q z)}\right)+\bar{N}\left(r, \frac{1}{P(f(z)) f(q z)-a(z)}\right) \\
& +S(r, f) \\
= & \bar{N}\left(r, \frac{1}{P(f(z)) f(q z)}\right)+S(r, f) \\
\leq & (m+1) T(r, f(z))+S(r, f)
\end{aligned}
$$

on a set of logarithmic density 1 . Taking using of the Valiron-Mohon'ko lemma and Lemma 2.5, we conclude that

$$
(n+1) T(r, f(z))=T(r, P(f(z)) f(z))+S(r, f)
$$




$$
\begin{aligned}
& =T(r, P(f(z)) f(q z))+S(r, f) \\
& \leq(m+1) T(r, f(z))+S(r, f)
\end{aligned}
$$

on a set of logarithmic density 1 , contradicting $n>m$. The proof of Theorem 2.1 is completed.

\section{Shared common values of $q$-difference polynomials}

Suppose that $f(z)$ and $g(z)$ are meromorphic functions, and $a \in \hat{\mathbb{C}}=\mathbb{C} \cup$ $\{\infty\}$. We say $f(z)$ and $g(z)$ share $a C M$ (counting multiplicities) if $f(z)-a$ and $g(z)-a$ have the same zeros with the same multiplicities. If $f(z)-a$ and $g(z)-a$ have the same zeros, we say $f(z)$ and $g(z)$ share $a I M$ (ignoring multiplicities).

Corresponding to the results on uniqueness in $[15,16]$, Zhang and Korhonen further obtained.

Theorem 3.A ([16, Theorem 5.1]). Let $f(z)$ and $g(z)$ be two transcendental meromorphic (resp. entire) functions of zero order. Suppose that $q$ is a nonzero complex constant and $n$ is an integer satisfying $n \geq 8$ (resp. $n \geq 4$ ). If $f(z)^{n} f(q z)$ and $g(z)^{n} g(q z)$ share $1, \infty C M$, then $f(z) \equiv \operatorname{tg}(z)$ for $t^{n+1}=1$.

Theorem 3.B ([16, Theorem 5.2]). Let $f(z)$ and $g(z)$ be two transcendental entire functions of zero order. Suppose that $q$ is a nonzero complex constant and $n \geq 6$ is an integer. If $f(z)^{n}(f(z)-1) f(q z)$ and $g(z)^{n}(g(z)-1) g(q z)$ share $1 C M$, then $f(z) \equiv g(z)$.

In this section, we firstly deduce more details about Theorem 3.A. Then, by combining all results above and the uniqueness of difference products on transcendental entire functions of finite order in [12], we further investigate the uniqueness of $q$-difference polynomials of meromorphic functions of zero order.

Theorem 3.1. Let $f(z)$ and $g(z)$ be two nonconstant meromorphic (resp. entire) functions of zero order. Suppose that $q$ is a nonzero complex constant and $n$ is an integer satisfying $n \geq 14$ (resp. $n \geq 6$ ). If $f(z)^{n} f(q z)$ and $g(z)^{n} g(q z)$ share $1 C M$, then $f(z) \equiv \operatorname{tg}(z)$ or $f(z) g(z)=t$, where $t^{n+1}=1$.

Remark 3.1. Under the assumption of Theorem 3.1, if $f(z)^{n} f(q z)$ and $g(z)^{n} g(q z)$ share $a \in \mathbb{C} \backslash\{0\} C M$, we also have $f(z) \equiv \operatorname{tg}(z)$ or $f(z) g(z)=t$, where $t^{n+1}=1$. In its proof, we only set

$$
F_{0}(z)=\frac{f(z)^{n} f(q z)}{a} \text { and } G_{0}(z)=\frac{g(z)^{n} g(q z)}{a} .
$$

Then $F_{0}(z)$ and $G_{0}(z)$ share $1 C M$. But the conclusion is not true if $a=0$. For example, let $f(z)=z$ and $g(z)=\frac{1}{2} z$. Then for all $q \neq 0, f(z)^{6} f(q z)=q z^{7}$ and $g(z)^{6} g(q z)=\frac{q}{2^{7}} z^{7}$ share $0 C M$. However, $f(z)=2 g(z), 2^{7} \neq 1$ and $f(z) g(z)=\frac{1}{2} z^{2}$. 
Theorem 3.2. Let $f(z)$ and $g(z)$ be two nonconstant meromorphic (resp. entire) functions of zero order. Suppose that $q$ is a nonzero complex constant and $n$ is an integer satisfying $n \geq 26$ (resp. $n \geq 12$ ). If $f(z)^{n} f(q z)$ and $g(z)^{n} g(q z)$ share 1 IM, then $f(z) \equiv \operatorname{tg}(z)$ or $f(z) g(z)=t$, where $t^{n+1}=1$.

Theorem 3.3. Let $f(z)$ and $g(z)$ be two nonconstant meromorphic (resp. entire) functions of zero order and $q$ be nonzero complex constant, and let $P(z)=a_{n} z^{n}+a_{n-1} z^{n-1}+\cdots+a_{1} z+a_{0}$ be a nonconstant polynomial with constant coefficients $a_{0}, a_{1}, \ldots, a_{n-1}, a_{n}(\neq 0)$, and $m$ be the number of the distinct zeros of $P(z)$. If $n>3 m+4$ (resp. $n>2 m+1)$ and $P(f(z)) f(q z)$ and $P(g(z)) g(q z)$ share $1, \infty C M$, then one of the following two results holds:

(1) $f(z) \equiv \operatorname{tg}(z)$ for a constant $t$ such that $t^{d}=1$, where $d=\operatorname{LCM}\left\{\lambda_{j}: j=\right.$ $0,1, \ldots, n\}$ denotes the lowest common multiple of $\lambda_{j}(j=0,1, \ldots, n)$, and

$$
\lambda_{j}=\left\{\begin{array}{l}
j+1, a_{j} \neq 0 \\
n+1, a_{j}=0
\end{array}\right.
$$

(2) $f(z)$ and $g(z)$ satisfy algebraic equation $R(f(z), g(z))=0$, where

$$
R\left(w_{1}, w_{2}\right)=P\left(w_{1}\right) w_{1}(q z)-P\left(w_{2}\right) w_{2}(q z) .
$$

In order to prove these theorems, we need some lemmas.

Lemma 3.1 ([15, Lemma 3]). Let $F(z)$ and $G(z)$ be two nonconstant meromorphic functions. If $F(z)$ and $G(z)$ share $1 C M$, one of the following three cases holds:

(1) $T(r, F(z)) \leq \bar{N}(r, F(z))+\bar{N}_{(2}(r, F(z))+\bar{N}(r, G(z))$

$$
\begin{aligned}
& +\bar{N}_{(2}(r, G(z))+\bar{N}\left(r, \frac{1}{F(z)}\right)+\bar{N}_{(2}\left(r, \frac{1}{F(z)}\right) \\
& +\bar{N}\left(r, \frac{1}{G(z)}\right)+\bar{N}_{(2}\left(r, \frac{1}{G(z)}\right)+S(r, F)+S(r, G),
\end{aligned}
$$

and similarly for $T(r, G(z))$;

(2) $F(z) \equiv G(z)$

(3) $F(z) G(z) \equiv 1$,

where $\bar{N}_{(2}\left(r, \frac{1}{F(z)}\right)=\bar{N}\left(r, \frac{1}{F(z)}\right)-N_{(1}\left(r, \frac{1}{F(z)}\right)$ and $N_{(1}\left(r, \frac{1}{F(z)}\right)$ is the counting function of the simple zeros of $F(z)$ in $\{z:|z| \leq r\}$.

Remark 3.2. Set

$$
N_{2}\left(r, \frac{1}{F(z)}\right)=\bar{N}\left(r, \frac{1}{F(z)}\right)+N_{(2}\left(r, \frac{1}{F(z)}\right) .
$$

Then we can find that $N_{2}\left(r, \frac{1}{F(z)}\right)$ denotes the counting function of zeros of $F(z)$ such that the simple zeros are counted once and the multiple zeros are counted twice, and the inequality (3.1) turns into

$$
T(r, F(z)) \leq N_{2}(r, F(z))+N_{2}\left(r, \frac{1}{F(z)}\right)+N_{2}(r, G(z))
$$




$$
+N_{2}\left(r, \frac{1}{G(z)}\right)+S(r, F)+S(r, G) .
$$

Lemma 3.2 ([13, Lemma 2.3]). Let $F(z)$ and $G(z)$ be two nonconstant meromorphic functions sharing the value 1 IM. Let

$$
H(z)=\left(\frac{F^{\prime \prime}(z)}{F^{\prime}(z)}-2 \frac{F^{\prime}(z)}{F(z)-1}\right)-\left(\frac{G^{\prime \prime}(z)}{G^{\prime}(z)}-2 \frac{G^{\prime}(z)}{G(z)-1}\right) .
$$

If $H(z) \not \equiv 0$, then

$$
\begin{aligned}
& T(r, F(z))+T(r, G(z)) \\
\leq & 2\left[N_{2}(r, F(z))+N_{2}(r, G(z))+N_{2}\left(r, \frac{1}{F(z)}\right)+N_{2}\left(r, \frac{1}{G(z)}\right)\right] \\
& +3\left[\bar{N}(r, F(z))+\bar{N}(r, G(z))+\bar{N}\left(r, \frac{1}{F(z)}\right)+\bar{N}\left(r, \frac{1}{G(z)}\right)\right] \\
& +S(r, F)+S(r, G) .
\end{aligned}
$$

In the follows, Theorems 3.1-3.3 will be proved.

Proof of Theorem 3.1. Let $F(z)=f(z)^{n} f(q z)$ and $G(z)=g(z)^{n} g(q z)$. Thus, $F(z)$ and $G(z)$ share $1 C M$. Suppose first that $F(z) \neq G(z)$ and $F(z) G(z) \neq 1$.

If $f(z)$ and $g(z)$ are meromorphic of zero order, then we deduce from the first main theorem and Lemma 2.4 that

$$
\begin{aligned}
n T(r, f(z))+S(r, f) & =T(r, P(f(z))) \\
& \leq T(r, P(f(z)) f(q z))+T\left(r, \frac{1}{f(q z)}\right)+S(r, f) \\
& \leq T(r, P(f(z)) f(q z))+T(r, f(z))+S(r, f) .
\end{aligned}
$$

Therefore

(3.3) $(n-1) T(r, f(z)) \leq T(r, P(f(z)) f(q z))+S(r, f)=T(r, F(z))+S(r, f)$.

Similarly,

$$
(n-1) T(r, g(z)) \leq T(r, G(z))+S(r, g) .
$$

By using Lemma 2.4 again, we also have

$$
\begin{aligned}
& T(r, F(z)) \leq(n+1) T(r, f(z))+S(r, f) \text { and } \\
& T(r, G(z)) \leq(n+1) T(r, g(z))+S(r, g) .
\end{aligned}
$$

Now, we conclude from Nevanlinna main theorems, Lemma 2.4 and (3.5) that

$$
\begin{aligned}
T(r, F(z)) & \leq \bar{N}(r, F(z))+\bar{N}\left(r, \frac{1}{F(z)}\right)+\bar{N}\left(r, \frac{1}{F-1}\right)+S(r, F) \\
& \leq \bar{N}(r, f(z))+\bar{N}(r, f(q z))+\bar{N}\left(r, \frac{1}{f(z)}\right)
\end{aligned}
$$




$$
\begin{aligned}
& +\bar{N}\left(r, \frac{1}{f(q z)}\right)+\bar{N}\left(\frac{1}{G-1}\right)+S(r, f) \\
\leq & 4 T(r, f(z))+T(r, G(z))+S(r, f) \\
\leq & 4 T(r, f(z))+(n+1) T(r, g(z))+S(r, f)+S(r, g) .
\end{aligned}
$$

Thus, the above inequality and (3.3) yield

$$
(n-5) T(r, f(z)) \leq(n+1) T(r, g(z))+S(r, f)+S(r, g) .
$$

Similarly,

$$
(n-5) T(r, g(z)) \leq(n+1) T(r, f(z))+S(r, f)+S(r, g) .
$$

It follows from Remark 3.2 and Lemma 2.4 that

$N_{2}\left(r, \frac{1}{F(z)}\right) \leq 2 \bar{N}\left(r, \frac{1}{f(z)}\right)+N\left(r, \frac{1}{f(q z)}\right)+S(r, f) \leq 3 T(r, f(z))+S(r, f)$.

Similarly, we also have

$$
\begin{aligned}
& N_{2}(r, F(z)) \leq 3 T(r, f(z))+S(r, f), \\
& N_{2}\left(r, \frac{1}{G(z)}\right) \leq 3 T(r, g(z))+S(r, g), \\
& N_{2}(r, G(z)) \leq 3 T(r, g(z))+S(r, g) .
\end{aligned}
$$

Therefore, (3.2), (3.5), (3.8)-(3.11) yield

$$
\begin{aligned}
T(r, F(z))+T(r, G(z)) \leq & 2 N_{2}(r, F(z))+2 N_{2}\left(r, \frac{1}{F(z)}\right)+2 N_{2}(r, G(z)) \\
& +2 N_{2}\left(r, \frac{1}{G(z)}\right)+S(r, F)+S(r, G) \\
\leq & 12[T(r, f(z))+T(r, g(z))]+S(r, f)+S(r, g) .
\end{aligned}
$$

Thus, we deduce from (3.3), (3.4) and (3.12) that

$$
(n-13)[T(r, f(z))+T(r, g(z))] \leq S(r, f)+S(r, g),
$$

contradicting $n \geq 14$.

If, on the other hand, $f(z)$ and $g(z)$ are entire of zero order. Replacing (3.3) and (3.4) by Lemma 2.5, and using the similar method above, we obtain

$$
(n-5)[T(r, f(z))+T(r, g(z))] \leq S(r, f)+S(r, g),
$$

contradicting $n \geq 6$.

So, by Lemma 3.1, we obtain either $F(z) \equiv G(z)$ or $F(z) G(z) \equiv 1$.

If $F(z) \equiv G(z)$, i.e., $f(z)^{n} f(q z)=g(z)^{n} g(q z)$, by denoting $h(z)=\frac{f(z)}{g(z)}$, we obtain

$$
h(z)^{n} h(q z)=1 .
$$


It follows from Lemma 2.4 and (3.13) that

$$
n T(r, h(z))=T\left(r, h(z)^{n}\right)=T\left(r, \frac{1}{h(q z)}\right) \leq T(r, h(z))+S(r, h) .
$$

Then $h(z)$ must be nonzero constant since $n \geq 6$. Suppose that $h(z)=t$, we deduce from (3.13) that $t^{n+1}=1$. Therefore, $f(z)=t g(z)$ and $t^{n+1}=1$.

If $F(z) G(z) \equiv 1$, i.e.,

$$
f(z)^{n} f(q z) g(z)^{n} g(q z)=1 .
$$

Set $s(z)=f(z) g(z)$. Then $s(z)^{n} s(q z)=1$. Similar to the discussion of (3.13), we also get $s(z)$ must be a nonzero constant, say $t$. Obviously, $t^{n+1}=1$ from (3.14). Therefore, $f(z) g(z)=t$ and $t^{n+1}=1$. The proof of Theorem 3.1 is completed.

Proof of Theorem 3.2. Let $F(z)=f(z)^{n} f(q z)$ and $G(z)=g(z)^{n} g(q z)$. Similar to the proof of Theorem 3.1, we still obtain that (3.3)-(3.11) hold. Let $H(z)$ be defined as Lemma 3.2 and suppose that $H(z) \not \equiv 0$.

If $f(z)$ and $g(z)$ are meromorphic of zero order, then we deduce from Lemma 2.4 that

(3.15) $\bar{N}(r, F(z)) \leq \bar{N}(r, f(z))+\bar{N}(r, f(q z))+S(r, f) \leq 2 T(r, f(z))+S(r, f)$.

Similarly,

$$
\begin{aligned}
& \bar{N}(r, G(z)) \leq 2 T(r, g(z))+S(r, g), \\
& \bar{N}\left(r, \frac{1}{F(z)}\right) \leq 2 T(r, f(z))+S(r, f), \\
& \bar{N}\left(r, \frac{1}{G(z)}\right) \leq 2 T(r, g(z))+S(r, g) .
\end{aligned}
$$

It follows from Lemma 3.2, (3.8)-(3.11) and (3.15)-(3.18) that

$$
T(r, F(z))+T(r, G(z)) \leq 24[T(r, f(z))+T(r, g(z))]+S(r, f)+S(r, g) .
$$

Therefore, we deduce from (3.3) and (3.4) and above inequality that $(n-1)[T(, f(z))+T(r, g(z))] \leq 24[T(r, f(z))+T(r, g(z))]+S(r, f)+S(r, g)$, contradicting $n \geq 26$.

If, on the other hand, $f(z)$ and $g(z)$ are entire of zero order, then, replacing (3.3) and (3.4) by Lemma 2.5, and using the similar method above, we also get

$$
(n-11)[T(r, f(z))+T(r, g(z))] \leq S(r, f)+S(r, g),
$$

contradicting $n \geq 12$.

Thus, using Lemma 3.2 again, we get $H(z) \equiv 0$, i.e.,

$$
\frac{F^{\prime \prime}(z)}{F^{\prime}(z)}-2 \frac{F^{\prime}(z)}{F(z)-1}=\frac{G^{\prime \prime}(z)}{G^{\prime}(z)}-2 \frac{G^{\prime}(z)}{G(z)-1} .
$$


By integrating the above equality twice, we conclude that

$$
F(z)=\frac{(b+1) G(z)+(a-b-1)}{b G(z)+(a-b)},
$$

where $a(\neq 0), b$ are two constants. In order to prove the conclusions of Theorem 3.2 are true, we will prove that either $F(z)=G(z)$ or $F(z) G(z)=1$. Now, according to the coefficients of $(3.19)$, we need to prove the following three cases.

Case 3.1. $b \neq 0,-1$.

If $a-b-1 \neq 0$, we obtain from (3.19) that

$$
\bar{N}\left(r, \frac{1}{F(z)}\right)=\bar{N}\left(r, \frac{1}{G(z)+\frac{a-b-1}{b+1}}\right) .
$$

Obviously, by Valiron-Mohon'ko lemma, (3.3), (3.4), (3.5) and (3.19) show that

$$
\left\{\begin{aligned}
(n-1) T(r, f(z)) & \leq(n+1) T(r, g)+S(r, f)+S(r, g), \\
(n-1) T(r, g(z)) & \leq(n+1) T(r, g)+S(r, f)+S(r, g) .
\end{aligned}\right.
$$

Thus, $S(r, f)=S(r, g)$.

Now, we may apply the second main theorem, Lemma 2.4, (3.4) and (3.20) to conclude that

$$
\begin{aligned}
(n-1) T(r, g(z)) \leq & T(r, G(z))+S(r, g) \\
\leq & \bar{N}(r, G(z))+\bar{N}\left(r, \frac{1}{G(z)}\right)+\bar{N}\left(r, \frac{1}{G(z)+\frac{a-b-1}{b+1}}\right) \\
& +S(r, g) \\
\leq & \bar{N}(r, G(z))+\bar{N}\left(r, \frac{1}{G(z)}\right)+\bar{N}\left(r, \frac{1}{F(z)}\right)+S(r, g) \\
\leq & \bar{N}(r, g(z))+\bar{N}(r, g(q z))+\bar{N}\left(r, \frac{1}{g(z)}\right)+\bar{N}\left(r, \frac{1}{g(q z)}\right) \\
& +\bar{N}\left(r, \frac{1}{f(z)}\right)+\bar{N}\left(r, \frac{1}{f(q z)}\right)+S(r, f)+S(r, g) \\
\leq & 2 T(r, f(z))+4 T(r, g(z))+S(r, g) \\
\leq & \left(\frac{2(n+1)}{n-1}+4\right) T(r, g)+S(r, g) .
\end{aligned}
$$

This implies that $n^{2}-8 n+3 \leq 0$, contradicting $n \geq 12$.

If $a-b-1=0$, then (3.19) turns out to be

$$
F(z)=\frac{(b+1) G(z)}{b G(z)+1} .
$$

Using a same method above, we also deduce a contradiction.

Case 3.2. $b=-1$ and $a \neq-1$. 
Otherwise, if $b=-1$ and $a=-1$, we obtain $F(z) G(z)=1$. Thus, we get $f(z) g(z)=t$ and $t^{n+1}=1$ by using similar proof of (3.14). So, we only need to prove it is incorrect if $b=-1$ and $a \neq-1$. Here, (3.19) turns into

$$
F(z)=\frac{a}{-G(z)+a+1} .
$$

Using a similar method of Case 3.1, we also deduce a contradiction.

Case 3.3. $b=0$ and $a \neq 1$.

(3.19) turns into

$$
F(z)=\frac{G(z)+a-1}{a} .
$$

Using a similar method of Case 3.1 again, we deduce a contradiction. Thus, $b=0$ and $a=1$. Therefore $F(z)=G(z)$. Similar to discuss (3.13), we deduce that $f(z)=\operatorname{tg}(z)$ and $t^{n+1}=1$. The Proof of Theorem 3.2 is completed.

Proof of Theorem 3.3. Since $P(f(z)) f(q z)$ and $P(g(z)) g(q z)$ share $1, \infty C M$, there exists an entire function $\alpha(z)$ such that

$$
\frac{P(f(z)) f(q z)-1}{P(g(z)) g(q z)-1}=e^{\alpha(z)}
$$

We deduce that $e^{\alpha(z)} \equiv$ constant, say $c$, since $f(z)$ and $g(z)$ are both meromorphic of zero order. Rewriting (3.22), we obtain

$$
c P(g(z)) g(q z)=P(f(z)) f(q z)-1+c .
$$

We assert that $c=1$.

If $c \neq 1, f(z)$ and $g(z)$ are meromorphic of zero order, then we may apply Nevanlinna main theorems, Lemma 2.2 and (3.23) to obtain

$$
\begin{aligned}
T(r, P(f(z) f(q z))) \leq & \bar{N}(r, P(f(z)) f(q z))+\bar{N}\left(r, \frac{1}{P(f(z)) f(q z)}\right) \\
& +\bar{N}\left(r, \frac{1}{P(f(z)) f(q z)-1+c}\right)+S(r, f) \\
\leq & \bar{N}(r, P(f(z)))+\bar{N}(r, f(q z))+\bar{N}\left(r, \frac{1}{P(f(z))}\right) \\
& +\bar{N}\left(r, \frac{1}{f(q z)}\right)+\bar{N}\left(r, \frac{1}{P(g(z)) g(q z)}\right)+S(r, f) \\
\leq & (m+1) \bar{N}(r, f(z))+(m+1) \bar{N}\left(r, \frac{1}{f(z)}\right) \\
& +\bar{N}\left(r, \frac{1}{P(g(z))}\right)+\bar{N}\left(r, \frac{1}{g(q z)}\right)+S(r, f)+S(r, g) \\
\leq & 2(m+1) T(r, f(z))+(m+1) T(r, g(z)) \\
& +S(r, f)+S(r, g) .
\end{aligned}
$$


We also deduce from the first main theorem and Lemma 2.4 that

$$
\begin{aligned}
n T(r, f(z))+S(r, f) & =T(r, P(f(z))) \\
& \leq T(r, P(f(z)) f(q z))+T\left(r, \frac{1}{f(q z)}\right)+S(r, f) \\
& \leq T(r, P(f(z)) f(q z))+T(r, f(z))+S(r, f) .
\end{aligned}
$$

Therefore

$$
(n-1) T(r, f(z)) \leq T(r, P(f(z)) f(q z))+O(1) .
$$

Substituting (3.24) into (3.25), we conclude that

$$
(n-2 m-3) T(r, f(z)) \leq(m+1) T(r, g(z))+S(r, f)+S(r, g) .
$$

Similarly,

$$
(n-2 m-3) T(r, g(z)) \leq(m+1) T(r, f(z))+S(r, f)+S(r, g) .
$$

By combining the last two inequalities, we get

$$
(n-3 m-4)[T(r, f(z))+T(r, g(z))] \leq S(r, f)+S(r, g),
$$

contradicting $n>3 m+4$.

If $c \neq 1, f(z)$ and $g(z)$ are entire of zero order, then

$$
\begin{aligned}
T(r, P(f(z)) f(q z)) \leq & \bar{N}\left(r, \frac{1}{P(f(z)) f(q z)}\right)+\bar{N}\left(r, \frac{1}{P(f(z)) f(q z)-1+c}\right) \\
& +S(r, f) \\
\leq & \bar{N}\left(r, \frac{1}{P(f(z)) f(q z)}\right)+\bar{N}\left(r, \frac{1}{P(g(z)) g(q z)}\right) \\
\leq & (m+1) T(r, f(z))+(m+1) T(r, g(z)) \\
& +S(r, f)+S(r, g) .
\end{aligned}
$$

Taking using of the Valiron-Mohon'ko lemma, Lemma 2.5 and above inequality, we deduce that

$$
\begin{aligned}
(n+1) T(r, f(z)) & =T(r, P(f(z)) f(z))+S(r, f) \\
& =T(r, P(f(z)) f(q z))+S(r, f) \\
& \leq(m+1) T(r, f(z))+(m+1) T(r, g(z))+S(r, f)+S(r, g) .
\end{aligned}
$$

Therefore,

$$
(n-m) T(r, f(z)) \leq(m+1) T(r, g(z))+S(r, f)+S(r, g) .
$$

Similarly,

$$
(n-m) T(r, g(z)) \leq(m+1) T(r, f(z))+S(r, f)+S(r, g) \text {. }
$$

(3.26) and (3.27) yield

$$
(n-2 m-1)[T(r, f(z))+T(r, g(z))] \leq S(r, f)+S(r, g),
$$

contradicting $n>2 m+1$. 
Thus, $c=1$ and (3.23) turns into

$$
P(f(z)) f(q z)=P(g(z)) g(q z) .
$$

Set $h(z)=\frac{f(z)}{g(z)}$. We will discuss the following two cases.

Case 3.A. Suppose that $h(z) \equiv$ constant, say $h$. Substituting $f(z)=h g(z)$ into (3.28), we obtain

$g(q z)\left[a_{n} g(z)^{n}\left(h^{n+1}-1\right)+a_{n-1} g(z)^{n-1}\left(h^{n}-1\right)+\cdots+a_{1} g(z)\left(h^{2}-1\right)+a_{0}(h-1)\right] \equiv 0$.

Since $g(z)$ is nonconstant meromorphic function, we have $g(q z) \not \equiv 0$. Hence, we get

$$
a_{n} g(z)^{n}\left(h^{n+1}-1\right)+a_{n-1} g(z)^{n-1}\left(h^{n}-1\right)+\cdots+a_{1} g(z)\left(h^{2}-1\right)+a_{0}(h-1) \equiv 0 .
$$

We assert that $h^{d}=1$, where $d$ is defined as the assumption of Theorem 3.3. Therefore, $f(z)=\operatorname{tg}(z)$ for a constant such that $t^{d}=1$. So, we need to prove the following two subcases.

Subcase 3.A.1. Suppose that $a_{n}$ is the only nonzero coefficient in (3.29). Since $g(z)$ is nonconstant meromorphic function, we obtain $h^{n+1}=1$.

Subcase 3.A.2. Suppose that $a_{n}$ is not the only nonzero coefficient in (3.29). If $h^{n+1} \neq 1$, by applying Valiron-Mohon'ko lemma to (3.29), we obtain $T(r, g(z))=S(r, g)$. This is a impossible. Hence, $h^{n+1}=1$. Similarly, we also deduce $h^{j+1}=1$ if $a_{j} \neq 0$ for $j=0,1, \ldots, n$.

Case 3.B. Suppose that $h(z)$ is not a constant. we deduce from (3.28) that $f(z)$ and $g(z)$ satisfy algebraic equation $R(f(z), g(z))=0$, where $R\left(w_{1}, w_{2}\right)=$ $P\left(w_{1}\right) w_{1}(q z)-P\left(w_{2}\right) w_{2}(q z)$

The proof of Theorem 3.3 is completed.

Acknowledgment. The author would like to thank the referees for their helpful remarks and suggestions to improve this paper. The author also thanks Professor Ilpo Laine and Professor Risto Korhonen for their hospitality during the visiting period in Department of Physics and Mathematics, University of Eastern Finland, and for their valuable suggestions to the present paper.

\section{References}

[1] D. C. Barnett, R. G. Halburd, W. Morgan, and R. J. Korhonen, Nevanlinna theory for the q-difference operator and meromorphic solutions of q-difference equations, Proc. Roy. Soc. Edinburgh Sect. A 137 (2007), no. 3, 457-474.

[2] Y. M. Chiang and S. J. Feng, On the Nevanlinna characteristic of $f(z+\eta)$ and difference equations in the complex plane, Ramanujan J. 16 (2008), no. 1, 105-129.

[3] Z. X. Chen, Value distribution of products of meromorphic functions and their differences, Taiwanese J. Math. 15 (2011), no. 4, 1411-1421.

[4] Z. X. Chen, Z. B. Huang, and X. M. Zheng, On properties of difference polynomials, Acta Math. Sci. Ser. B Engl. Ed. 31 (2011), no. 2, 627-633.

[5] R. G. Halburd and R. J. Korhonen, Difference analogue of the lemma on the logarithmic derivative with applications to difference equations, J. Math. Anal. Appl. 314 (2006), no. $2,477-487$. 
[6] - Nevanlinna theory for the difference operator, Ann. Acad. Sci. Fenn. Math. 31 (2006), no. 2, 463-478.

[7] W. K. Hayman, Meromorphic Functions, Clarendon Press, Oxford, 1964.

[8] J. Heittokangas, R. Korhonen, I. Laine, J. Rieppo, and J. Zhang, Value sharing results for shifts of meromorphic functions, and sufficient condition for periodicity, J. Math. Anal. Appl. 355 (2009), no. 1, 352-363.

[9] I. Laine and C. C. Yang, Clunie theorems for difference and q-difference polynomials, J. Lond. Math. Soc. (2) 76 (2007), no. 3, 556-566.

[10] Value distribution of difference polynomials, Proc. Japan Acad. Ser. A Math. Sci. 83 (2007), no. 8, 148-151.

[11] X. D. Luo and W. C. Lin, Value sharing results for shifts of meromorphic functions, J. Math. Anal. Appl. 377 (2011), no. 2, 441-449.

[12] X. G. Qi, K. Liu, and L. Z. Yang, Value sharing results of a meromorphic function $f(z)$ and $f(q z)$, Bull. Korean Math. Soc. 48 (2011), no. 6, 1235-1243.

[13] J. F. Xu and H. X. Yi, Uniqueness of entire functions and differential polynomials, Bull. Korean Math. Soc. 44 (2007), no. 4, 623-629.

[14] L. Yang, Value Distribution Theory and New Research (in Chinese), Science Press, Beijing, 1982.

[15] C. C. Yang and X. H. Hua, Uniqueness and value sharing of meromorphic functions, Ann. Acad. Sci. Fenn. Math. 22 (1997), no. 2, 395-406.

[16] J. L. Zhang and R. Korhonen, On the Nevanlinna characteristic of $f(q z)$ and its application, J. Math. Anal. Appl. 369 (2010), no. 2, 537-544.

School of Mathematical Sciences

South China Normal University

Guangzhou 510631, P. R.China

AND

Department of Physics and Mathematics

UNIVERSITY OF EASTERN FinLAND

P.O. Box 111, 80101, Joensuu, Finland

E-mail address: hzbo20019@sina.com, huangzhibo@scnu.edu.cn 